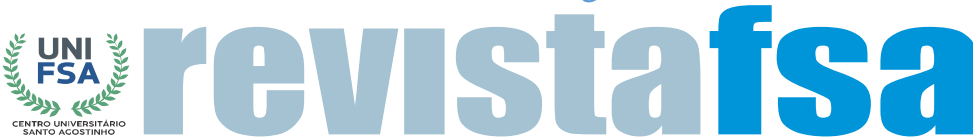 \\ www4.fsanet.com.br/revista
}

Rev. FSA, Teresina, v. 18, n. 6, art. 9, p. 169-185, jun. 2021 ISSN Impresso: 1806-6356 ISSN Eletrônico: 2317-2983 http://dx.doi.org/10.12819/2021.18.6.9

\section{Aplicação de Redes Neurais na Previsão de Custos de Produção de Frangos de Corte no Estado do Paraná}

\section{Application of Neural Networks to Forecast Broiler Production Cost in the State of Paraná}

José Airton Azevedo dos Santos

Doutor em Engenharia Elétrica pela Universidade Federal de Santa Catarina Professor Permanente do Programa de Pós-Graduação em Tecnologias Computacionais para o Agronegócio

E-mail: airton@utfpr.edu.br

Endereco: José Airton Azevedo dos Santos CÂMPUS MEDIANEIRA - Avenida Brasil, 4232 CEP 85884-000 - Caixa Postal 271 - Medianeira - PR - Brasil
Editor-Chefe: Dr. Tonny Kerley de Alencar Rodrigues

Artigo recebido em 23/05/2021. Última versão recebida em 01/06/2021. Aprovado em 02/06/2021.

Avaliado pelo sistema Triple Review: a) Desk Review pelo Editor-Chefe; e b) Double Blind Review (avaliação cega por dois avaliadores da área).

Revisão: Gramatical, Normativa e de Formatação 


\title{
RESUMO
}

A avicultura é um dos principais componentes do agronegócio paranaense. A produção de frangos de corte contribui significativamente para a economia estadual. $O$ produtor paranaense, na gestão da produção de frangos de corte, leva em conta os dados de desempenho, a forma de renumeração dos lotes e os custos de produção. Os custos de produção refletem diretamente na rentabilidade do seu negócio. Neste contexto, este trabalho tem como objetivo avaliar a eficácia de modelos de redes neurais artificiais, para previsão dos custos de produção de frangos de corte no estado do Paraná. A base de dados disponibilizada pela Empresa Brasileira de Pesquisa Agropecuária (EMBRAPA), apresenta uma série de custos mensal compreendida entre Janeiro/2010 e Novembro/2020. Modelos de previsão, baseados em Redes Neurais MLP (Multilayer Perception) e LSTM (Long Short-Term Memory) foram implementados, na linguagem Python, utilizando o framework Keras. Os resultados obtidos para um horizonte de onze meses, mostram que o modelo LSTM apresentou, na previsão dos custos de produção, um melhor desempenho.

Palavras-chave: Avicultura. Redes Neurais. Paraná.

\begin{abstract}
Broiler sector is one of the main components of agribusiness in Paraná. The production of broiler chickens contributes significantly to the state economy. The producer from Paraná, when managing the production of broiler chickens, takes into account the performance data, the form of renumbering the lots and the production costs. Production costs directly reflect the profitability of your business. In this context, this work aims to evaluate the effectiveness of models, of artificial neural networks, for forecasting the production costs of broilers in the state of Paraná. The database, made available by the Brazilian Agricultural Research Corporation (EMBRAPA), presents a series of monthly costs between January/2010 and November/2020. Prediction models, based on MLP (Multilayer Perception) and LSTM (Long Short-Term Memory) Neural Networks were implemented, in the Python language, using the Keras framework. The results obtained, for an eleven-month horizon, show that the LSTM model presented, in the forecast of production costs, a better performance.
\end{abstract}

Keywords: Broiler sector. Neural Networks. Paraná. 


\section{INTRODUÇÃO}

A avicultura é um dos principais componentes do agronegócio paranaense e brasileiro. Seu desenvolvimento pode ser considerado como o símbolo do crescimento e da modernização do setor (ABPA, 2020, PINHEIRO et al. 2020).

Os três estados da região sul (Rio Grande do Sul (RS), Santa Catarina (SC) e Paraná (PR)) são os principais produtores de frango de corte no Brasil. Segundo a AviSite (2020), 58,4\% ( $\mathrm{R} \$ 36,67$ bilhões) do valor bruto de produção agrícola vieram da região sul. A liderança absoluta é do estado do Paraná, cuja receita de 2019 com o frango foi estimada em pouco mais de R 20 bilhões. Seguem-no, na segunda e terceira posições, Santa Catarina e Rio Grande do Sul, com, respectivamente, $13,9 \%$ e 12,3\% do total.

Diversos fatores contribuíram para que o setor alcançasse o patamar atual, mas o fator preponderante foi o progresso técnico e tecnológico (JÚNIOR et al., 2009). Segundo Procópio et al. (2020), as inovações tecnológicas, nas áreas de genética, nutrição, sanidade e no processo produtivo, visaram pricipalmente o aumento da produtividade e o ganho financeiro.

Conhecer os custos de produção, em tempos de alta competitividade, não é dever somente das empresas que atuam no mercado de carnes, mas também do avicultor (SANDI; MIELE, 2012). Portanto, a previsão futura dos custos de produção, ajuda os produtores/empresas a estabelecerem vantagens competitivas para os seus negócios. Modelos de redes neurais artificiais têm sido muito utilizados, como ferramentas de previsão, em diversas áreas de aplicação. Algumas dessas aplicações incluem contabilidade, economia, mercado financeiro, ramo imobiliário, engenharia, entre outras. As redes neurais são ferramentas de grande importância em um sistema de planejamento, pois direcionam a melhor forma de aproveitar todos os recursos disponíveis (PINHEIRO et al. 2020).]

Segundo Haykin (2001) uma Rede Neural Artificial (RNA) é um processador paralelamente distribuido composto por unidades de processamento simples que possuem a habilidade de apreender padrões complexos de dados e generalizar a informação apreendida. Sua aplicação se dá para problemas de classificação e problemas de predição, tanto para dados transversos (regressão) quanto para dados de séries temporais (GOMES, 2005). É uma técnica de modelagem que pode resolver muitos problemas não lineares e complexos (SEBASTIAN, 2016; BASTIANI et al., 2018; LIMA et al., 2009; MUNTASER et al., 2017).

Diversos trabalhos utilizaram métodos de previsão em aplicações voltadas à avicultura. Dentre eles, podem-se citar os trabalhos de: Pinheiro et al. (2020) que aplicaram redes LSTMs na previsão do preço do quilo de frango congelado e resfriado. Bastiani et al. 
(2018) que aplicaram redes neurais MLPs para predição e classificação, de variáveis produtivas, das aves produzidas por uma cooperativa localizada na região oeste paranaense. Johansen et al. (2019) que realizaram, por meio de redes neurais artificiais, a previsão do peso de abate de frangos de corte e Amrael et al. (2017) que também analisaram, po meio de redes neurais artificiais e visão de máquina, o peso de frangos de corte.

Embora a produção de frangos de corte tenha grande importância, para o agonegócio brasileiro, poucos trabalhos utilizam redes neurais na previsão dos custos de produção dos frangos, principalmente utilizando a biblioteca Keras.

Neste contexto, este trabalho tem como objetivo avaliar a eficácia de modelos, de redes neurais artificiais, para previsão dos custos de produção de frangos no estado do Paraná, no período entre janeiro de 2010 e Novembro de 2020.

$\mathrm{O}$ artigo é organizado da seguinte maneira. Na Seção Fundamentação Teórica as redes neurais utilizadas neste trabalho são apresentadas. Na Seção Materiais e Métodos é apresentada a metodologia utilizada na previsão dos custos de produção de frangos de corte. Na Seção Resultados e Discussão são apresentados os resultados obtidos e suas respectivas discussões. Comentários finais, conclusões e perspectivas de trabalhos futuros, na Seção Considerações Finais, finalizam o artigo.

\section{REFERENCIAL TEÓRICO}

Nesta seção apresentam-se as redes neurais abordadas nesta pesquisa, bem como as métricas utilizadas.

\subsection{Redes Neurais}

Neste trabalho utilizaram-se, como métodos de previsão, as Redes Neurais Artificiais MLP e LSTM apresentadas a seguir:

\section{Multilayer Perception (MLP):}

As redes MLPs representam uma classe de redes neurais utilizadas na modelagem de sistemas complexos. Sendo muito utilizadas na previsão de dados financeiros, porque podem aproximar muito bem funções não lineares. As redes são compostas por uma camada de entrada, uma ou mais camadas ocultas de unidades de processamento e uma camada de saída. Seu treinamento é supervisionado e utiliza o algoritmo backpropagation. O algoritmo backpropagation é uma das ferramentas mais utilizadas para o treinamento de Redes Neurais 
Artificiais. As redes MLPs podem realizar tanto regressão quanto classificação (SEBASTIAN, 2016; BASTIANI et al., 2018).

Long Short-Term Memory (LSTM):

As redes LSTMs são redes neurais recorrentes capazes de apreender dependências a longo prazo. São adequadas para classificar, processar e fazer previsões com base em dados de séries temporais. Segundo Zao (2017), operam com muito boa precisão em uma grande variedade de problemas.

A topologia de um neurônio, de uma rede LSTM, é baseada em uma célula de memória (Figura 1). Uma célula de memória possui um componente de memória para armazenar sequências recentes, o que o torna mais inteligente que um neurônio clássico. As redes LSTMs utilizam portas que permitem ajustes de peso. Estas portas têm a capacidade de modificar (adicionar, descartar ou atualizar) as informações no tempo, de modo a prever melhor estados futuros (GRAVES, 2014, NELSON et al., 2017; MESQUITA et al., 2020).

Figura 1 - Célula LSTM

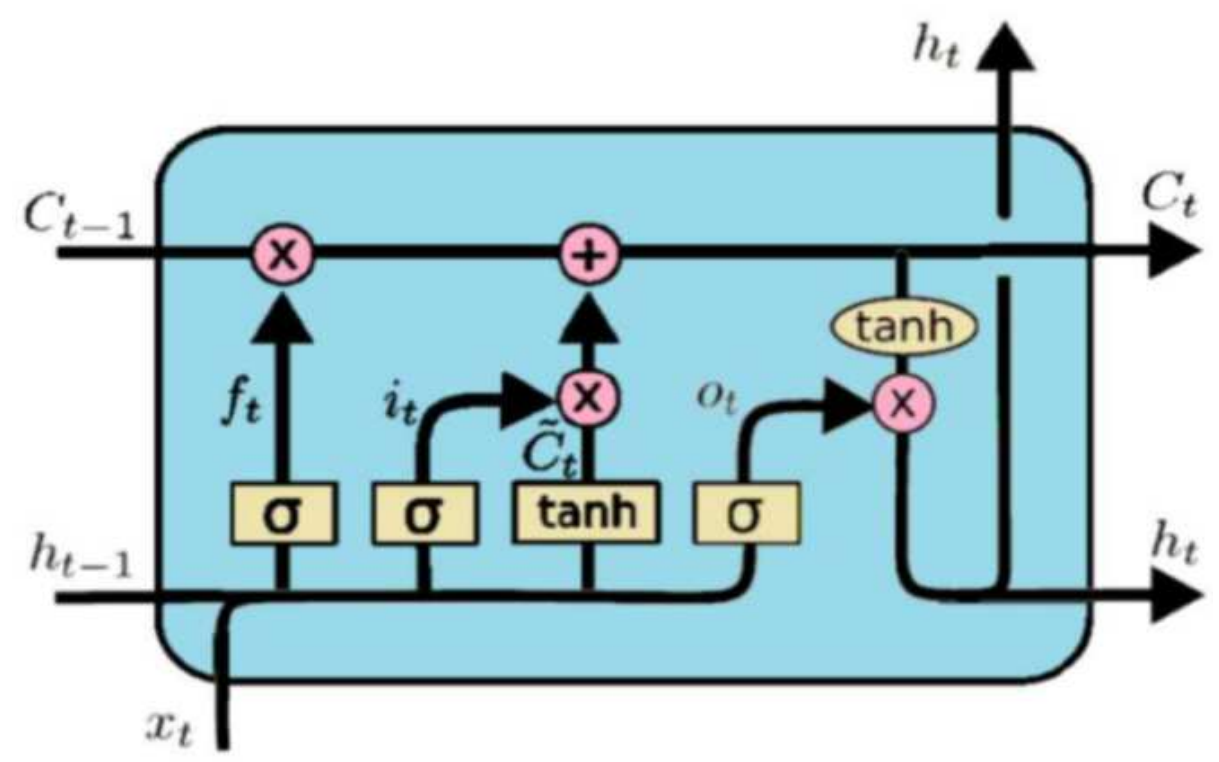

Fonte: Chevaller (2018).

$\mathrm{Na}$ arquitetura da célula LSTM tem-se 3 portas (porta de esquecimento $\mathrm{f}_{\mathrm{t}}$, porta de entrada $i_{t}$ e a porta de saída $O_{t}$ ). A porta de entrada pode determinar como a sequência de entrada $\mathrm{x}_{\mathrm{t}}$ altera o estado de memória da célula. A porta de saída determina o que produzir com base na entrada e na memória da célula. A porta de esquecimento determina quais informações devem ser jogadas fora pela célula (GRAVES, 2014). 


\subsection{Métricas}

Neste trabalho, os modelos, de redes neurais artificiais MLP e LSM, foram avaliados pelas seguintes métricas (CANKURT; SUBASI, 2015; PINHEIRO et al., 2020).

Coeficiente de Determinação $\left(r^{2}\right)$ :

A qualidade de ajuste de um modelo pode ser avaliada pelo coeficiente de determinação. Este coeficiente indica quanto o modelo foi capaz de explicar os dados coletados. Valor perfeito $-\mathrm{r}^{2}=1$.

Raiz Quadrada do Erro Médio Quadrático (RMSE):

Raiz do erro médio quadrático da diferença entre a predição e o valor real. Tem a vantagem de ser expressa na mesma unidade de medida da variável estudada. Valor perfeito RMSE $=0$.

Erro Médio Absoluto (MAE):

Como o RMSE, o MAE possui dimensão igual à dimensão dos valores observados e preditos. Seu valor representa o desvio médio entre observado e predito. Comparando o RMSE e o MAE, o primeiro dá um peso maior para desvios grandes (pois são elevados ao quadrado), enquanto o MAE dá um peso igual a todos os desvios. Valor perfeito - MAE=0.

\section{METODOLOGIA}

A metodologia utilizada, neste estudo, pode ser classificada quanto à natureza, objetivos e à forma de abordar o problema. A classificação quanto à natureza pode ser considerada aplicada, quanto aos objetivos descritiva e quanto à forma de abordar o problema quantitativa (GIL, 2002).

\subsection{Base de Dados}

Para previsão dos custos de produção de frangos, em Aviário Climatizado Positivo ( $\mathrm{R} \$ / \mathrm{kg}$ vivo), utilizou-se uma base de dados, com 131 meses (Janeiro/2010 a Novembro/2020), disponibilizada pela Empresa Brasileira de Pesquisa Agropecuária em seu site (EMBRAPA, 2020). Os dados coletados, do site da EMBRAPA, já estavam limpos e sem a presença de outliers. Portanto, os dados que compõem a amostra de pesquisa não sofrerão qualquer tipo de tratamento.

Os dez primeiros registros, do conjunto de dados da EMBRAPA, são apresentados na Tabela 1. 
Tabela 1 - Dez primeiros registros do arquivo da EMBRAPA

\begin{tabular}{cc}
\cline { 2 - 2 } DATA & $\begin{array}{c}\text { CUSTOS } \\
(\mathbf{R} \mathbf{\$} / \mathbf{k g})\end{array}$ \\
\hline $2010-1$ & 1,29 \\
$2010-2$ & 1,22 \\
$2010-3$ & 1,21 \\
$2010-4$ & 1,23 \\
$2010-5$ & 1,26 \\
$2010-6$ & 1,25 \\
$2010-7$ & 1,4 \\
$2010-8$ & 1,48 \\
$2010-9$ & 1,56 \\
$2010-10$ & 1,62 \\
\hline
\end{tabular}

A série histórica, do custo de produção de frangos de corte, no estado do Paraná, é apresentada na Figura 2. Pode-se notar, por meio desta figura, uma tendência ao aumento do custo de produção do frango ao longo dos anos.

Figura 2 - Série: Custo de produção de frangos

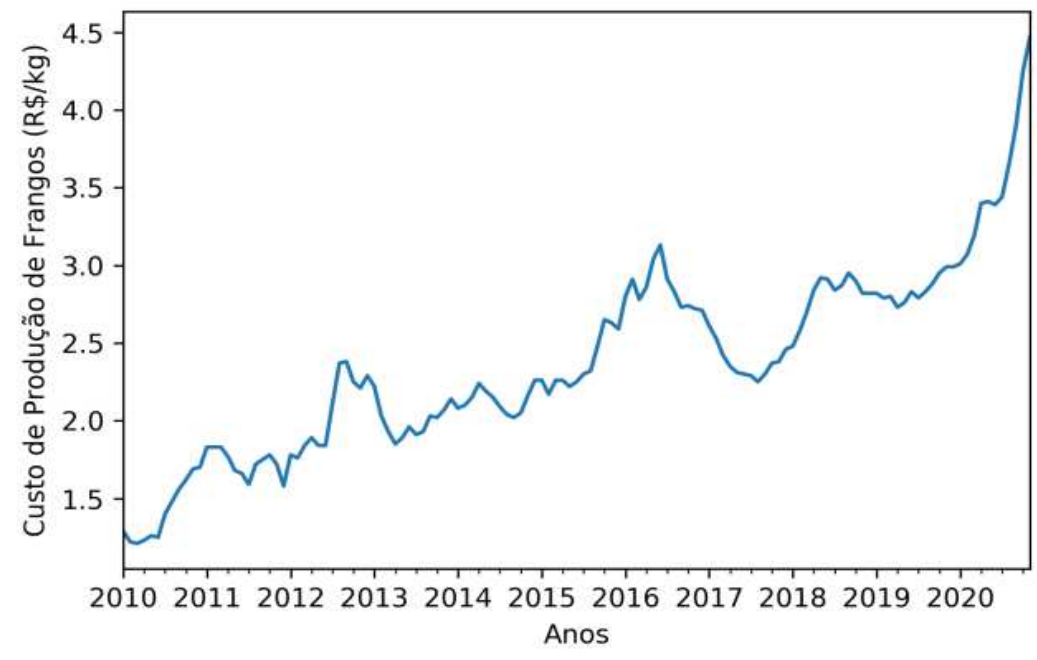

Fonte: Os autores.

\subsection{Etapas do Trabalho}

O trabalho foi dividido em quatro etapas: Coleta e análise dos dados, Modelagem, Teste e Previsão (Quadro 1). 


\begin{tabular}{|c|c|}
\hline ETAPAS & DESCRIÇÃO \\
\hline $\begin{array}{c}\text { Coleta e análise dos } \\
\text { dados }\end{array}$ & $\begin{array}{l}\text { Nesta etapa os dados foram coletados e } \\
\text { analisados por meio de uma análise } \\
\text { exploratória. A análise exploratória } \\
\text { ajuda a extrair informações importantes } \\
\text { de um conjunto de dados. }\end{array}$ \\
\hline Modelagem & $\begin{array}{l}\text { Na etapa de modelagem foram } \\
\text { implementados vários modelos de redes } \\
\text { neurais MLP e LSTM. Nesta etapa os } \\
\text { modelos com melhor desempenho, no } \\
\text { conjunto de validação, foram } \\
\text { selecionados. Observa-se que os } \\
\text { modelos de séries temporais, do custo } \\
\text { de produção de frango, incluem uma } \\
\text { camada de entrada, uma camada oculta } \\
\text { e uma camada de saída. }\end{array}$ \\
\hline Teste & $\begin{array}{l}\text { Nesta etapa os modelos foram testados } \\
\text { com dados que não participaram do } \\
\text { processo de treinamento e validação. }\end{array}$ \\
\hline Previsão & $\begin{array}{l}\text { Finalmente, nesta etapa, realizou-se a } \\
\text { previsão do custo de produção de } \\
\text { frangos para os meses de } \\
\text { novembro/2020 à Junho/2021. }\end{array}$ \\
\hline
\end{tabular}

Fonte: Os autores.

\subsection{Treinamento, Validação e Teste}

As 131 observações da base de dados da EMBRAPA foram utilizadas na criação dos subconjuntos de treinamento, validação e de teste. A tarefa de um modelo de machine learning é prever dados que não foram utilizados em sua construção. Portanto, os custos relativos aos meses de Janeiro/2020 a Novembro/2020 foram retirados do conjunto de dados, para serem utilizados posteriormente no teste do modelo (conjunto de teste).

Neste trabalho utilizou-se o método de fragmentação de Houldout, no qual a base de dados, sem o conjunto de teste, foi dividida com $67 \%$ (80) dos dados para treinamento dos algoritmos (Conjunto de Treinamento) e 33\% (40) para validação (Conjunto de validação).

\subsection{Critério de Parada}

Definiu-se, como critério de parada do treinamento, a função EarlyStopping() com o parâmetro patience $=25$. O parâmetro patience indica o número de épocas, após o qual, 
nenhuma melhoria foi observada. Segundo Silva (2020), este método ajuda a projetar uma rede neural com bom poder de generalização.

\subsection{Recursos}

Para implementação dos códigos, em Python 3, utilizou-se o ambiente Jupyter Notebook. Os modelos foram implementados por meio da biblioteca Keras. Keras é uma biblioteca para prototipagem rápida e fácil de redes neurais. Roda como frontend em TensorFlow ou Theano. TensorFlow é uma plataforma de código aberto para computação numérica e aprendizado de máquina (PINHEIRO et al., 2020).

\section{RESULTADOS E DISCUSSÕES}

Inicialmente, neste trabalho, realizou-se uma análise descritiva dos dados (Tabela 2).

\section{Tabela 2 - Análise descritiva de dados - EMBRAPA}

\begin{tabular}{lc}
\hline Observações & 131 \\
Média $(\mathrm{R} \$ \mathrm{~kg})$ & 2,36 \\
Mínimo $(\mathrm{R} \$ / \mathrm{kg})$ & 1,21 \\
Máximo $(\mathrm{R} \$ \mathrm{~kg})$ & 4,47 \\
Desvio Padrão (R $\$ / \mathrm{kg})$ & 0,60 \\
Coeficiente de Variação & 25,4 \\
\hline
\end{tabular}

Fonte: Os autores.

Pode-se observar, dos dados apresentados na Tabela 2, que o custo, para o período em estudo, ficou em média de 2,36 R $\$ / \mathrm{kg}$. O maior custo foi de $4,47 \mathrm{R} \$ / \mathrm{kg}$ no mês de novembro de 2020, enquanto o menor foi de $1,21 \mathrm{R} \$ / \mathrm{kg}$ no mês de março de 2010.

Observa-se também, da Tabela 2, que o coeficiente de variação é $25,4 \%$, considerado alto, o que indica variabilidade dos dados. Segundo Pimentel (2009), se o coeficiente de variação for inferior a $10 \%$ tem-se um coeficiente de variação baixo, de 10 a $20 \%$ médio, de 20 a $30 \%$ alto e acima de $30 \%$ muito alto.

O custo médio mensal de produção de frangos de corte, durante o período estudado é apresentado na Figura 3. Pode-se notar, por meio desta figura, que o custo médio de produção manteve-se praticamente constante durante os meses de Janeiro a Julho, tendo um pequeno aumento para os meses de Agosto a Dezembro. Observou-se um custo médio mínimo de 2,17 $\mathrm{R} \$ / \mathrm{kg}$ no mês Fevereiro, e um custo médio máximo de $2,53 \mathrm{R} \$ / \mathrm{kg}$ para o mês de Novembro. 
Figura 3 - Custo médio mensal da produção de frango

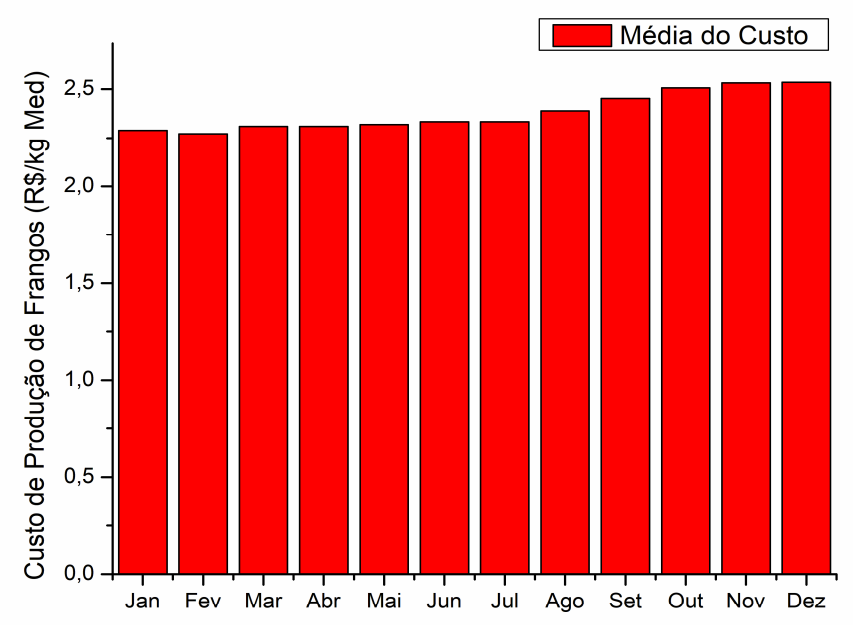

Fonte: Os autores.

\subsection{Treinamento e Validação}

Para escolha dos melhores modelos para previsão de custo de produção de frangos de corte, vários modelos LSTM e MLP foram treinados. Os modelos com melhor desempenho utilizaram os seguintes parâmetros:

Tabela 3 - Indicadores de desempenho

\begin{tabular}{lc}
\hline Número de Neurônios - Camada oculta & 8 \\
Número de Neurônios - Camada de saída & 1 \\
Função de Ativação - Camada oculta & Relu \\
Função de Ativação - Camada de saída & Linear \\
algoritmo de otimização & Adam \\
Batch & 30 \\
Learning rate & 0,0009 \\
\hline
\end{tabular}

Fonte: Os autores.

Os indicadores de desempenho, dos modelos LSTM e MLP, são apresentados na Tabela 4.

Tabela 4 - Indicadores de desempenho

\begin{tabular}{cccccccc}
\cline { 2 - 7 } & \multicolumn{3}{c}{ Treino } & \multicolumn{3}{c}{ Validação } \\
\cline { 2 - 7 } LSTM & $\mathbf{r}^{\mathbf{2}}$ & RMSE & MAE & $\mathbf{r}^{\mathbf{2}}$ & RMSE & MAE \\
\cline { 2 - 7 } MLP & 0,948 & 0,092 & 0,071 & 0,925 & 0,063 & 0,051 \\
& 0,950 & 0,090 & 0,072 & 0,932 & 0,060 & 0,051 \\
\hline
\end{tabular}

Fonte: Os autores. 
Observa-se, dos dados apresentados na Tabela 4, que os resultados obtidos pelos modelos, para os conjuntos de treino e validação, possuem erros (RMSE e MAE) próximos de zero e coeficientes de determinação próximos de um. Destaca-se também que os resultados dos dois modelos são muito similares.

Na Figura 4 apresentam-se as curvas de aprendizagem dos modelos MLP e LSTM. Pode-se observar, nesta figura, a boa estabilidade na convergência das curvas de treino e validação para os dois modelos.

\section{Figura 4 - Curvas de aprendizagem - MLP e LSTM}
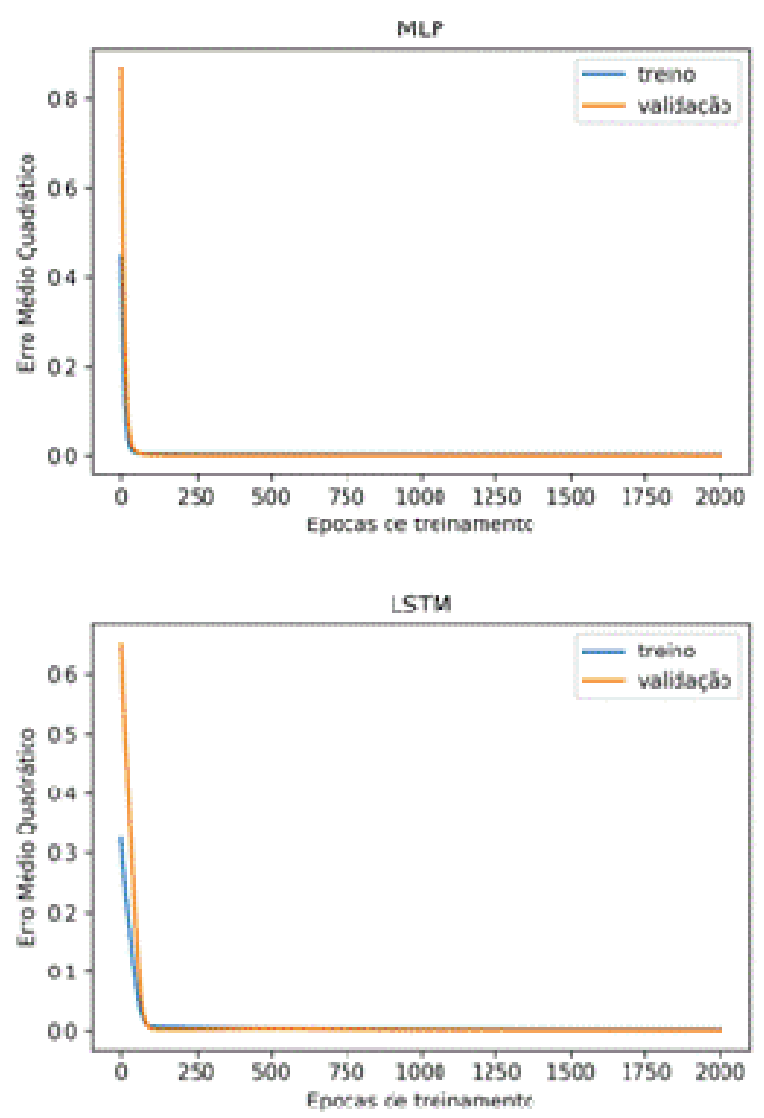

Fonte: Os autores.

Na sequência, avaliou-se, para o conjunto de dados de validação, a dispersão dos valores preditos em relação aos valores observados (Figura 5). Pode-se observar, dos gráficos, que os dados reais observados estão próximos à linha de ajuste. 
Figura 5 - Dados preditos em função dos dados observados
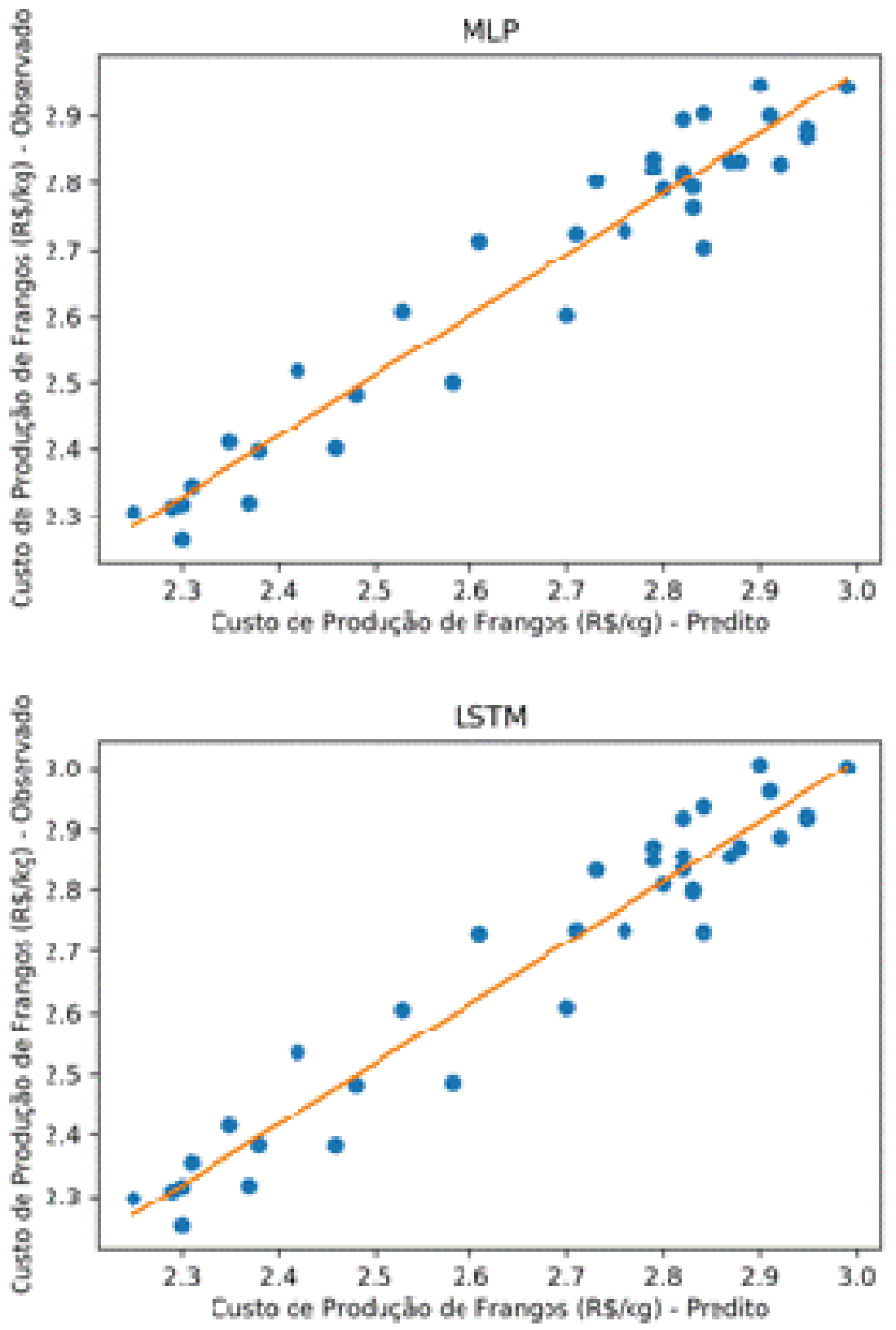

Fonte: Os autores.

Pode-se também observar, por meio da Figura 6, a boa aderência dos dados previstos com os dados reais para os dois modelos. 
Figura 6 - Custos de produção de frangos - Treino e Validação
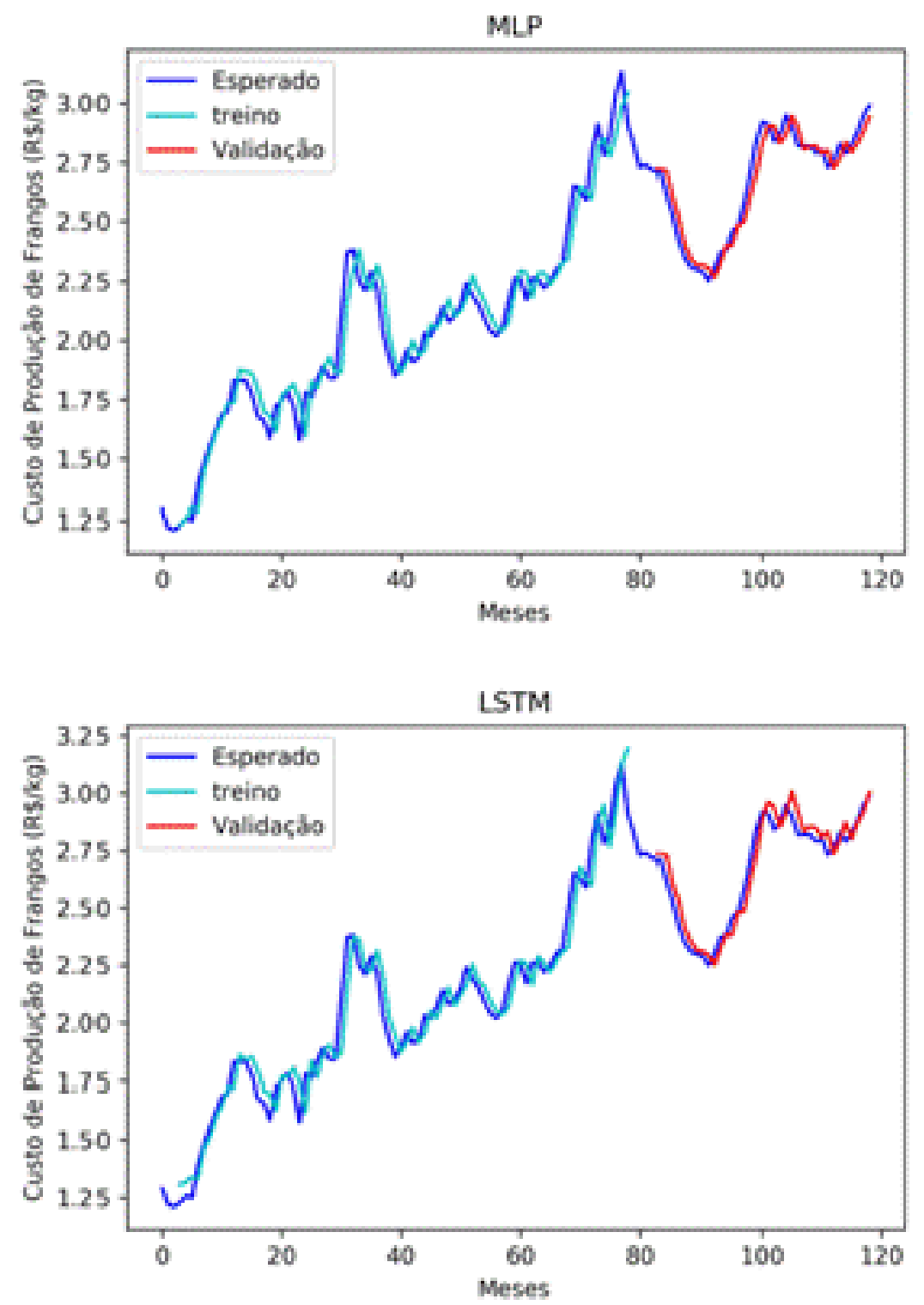

Fonte: Os autores.

\subsection{Teste e Previsões}

Na Tabela 5, apresentam-se os dados observados, preditos e os Erros Relativos Percentuais (ERP) para os onze meses de 2020, que não participaram da etapa de treinamento e teste. O ERP é obtido por meio da equação:

$$
E R P=\left|\frac{\text { Observado }- \text { Prodito }}{\text { Observado }}\right| \times 100
$$




\section{Tabela 5 - Custo da produção (Observados e Preditos $(\mathrm{R} \$ / \mathbf{k g}))$ e Erros percentuais relativos $(\%)$ )}

\begin{tabular}{cccccc}
\hline $\begin{array}{c}\text { Mê } \\
\text { s }\end{array}$ & $\begin{array}{c}\text { EMBRA } \\
\text { PA }\end{array}$ & MLP & $\begin{array}{c}\text { LST } \\
\text { M }\end{array}$ & $\begin{array}{c}\text { ERP- } \\
\text { MLP }\end{array}$ & $\begin{array}{c}\text { ERP- } \\
\text { LSTM }\end{array}$ \\
\hline Jan & 3,01 & 2,98 & 3,03 & $0,90 \%$ & $0,66 \%$ \\
Fev & 3,07 & 2,98 & 3,09 & $3,00 \%$ & $0,62 \%$ \\
Ma & & & & & \\
r & 3,19 & 2,97 & 3,16 & $6,77 \%$ & $1,10 \%$ \\
Abr & 3,40 & 2,97 & 3,23 & $12,68 \%$ & $5,00 \%$ \\
Mai & 3,41 & 2,97 & 3,32 & $13,05 \%$ & $2,70 \%$ \\
Jun & 3,39 & 2,96 & 3,42 & $12,65 \%$ & $0,94 \%$ \\
Jul & 3,44 & 2,96 & 3,55 & $14,04 \%$ & $3,11 \%$ \\
Ag & & & & & \\
o & 3,65 & 2,95 & 3,70 & $19,07 \%$ & $1,40 \%$ \\
Set & 3,90 & 2,95 & 3,90 & $24,33 \%$ & $0,13 \%$ \\
Out & 4,25 & 2,95 & 4,15 & $30,66 \%$ & $2,45 \%$ \\
No & & & & & \\
v & 4,47 & 2,94 & 4,48 & $34,14 \%$ & $0,29 \%$ \\
\hline \multicolumn{5}{c}{ Médi } \\
\cline { 3 - 5 } & & & $15,57 \%$ & $1,67 \%$ \\
\hline
\end{tabular}

Fonte: Os autores.

Por meio dos resultados apresentados, na Tabela 5, conclui-se que as previsões do modelo LSTM estão muito mais próximas dos valores fornecidos pela EMBRAPA, que as previsões do modelo MLP. Observa-se também, que o modelo LSTM apresenta um erro percentual relativo médio bem menor que o modelo MLP (ERP-LSTM=1,67\% e ERP-MLP= 15,57\%). Os resultados das previsões, em termos gráficos, são apresentados na Figura 7.

Figura 7 - Previsão dos custos de produção (Conjunto de Teste)

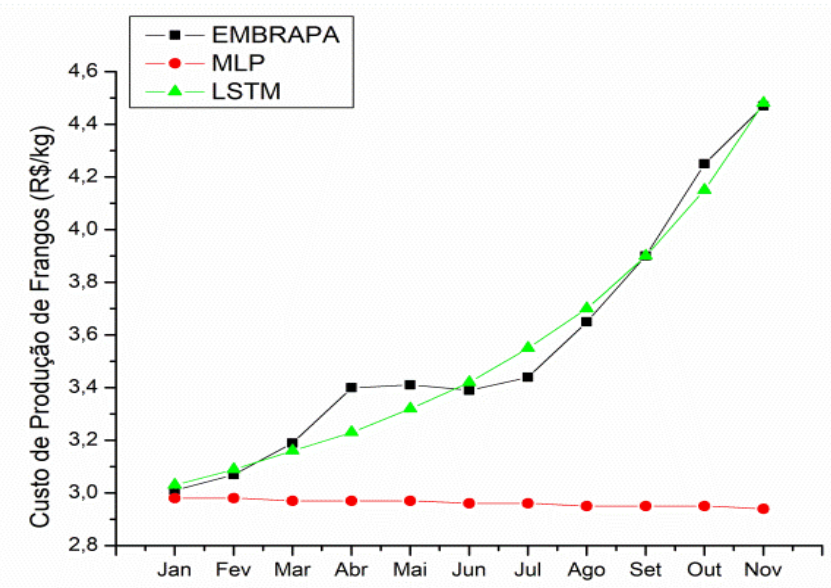

Fonte: Os autores. 
Na Figura 8 apresentam-se as previsões do custo de produção de frangos para os meses de Dezembro/2020 a Junho/2021.

Figura 8 - Previsões de Dez/2020 à Jun/2021

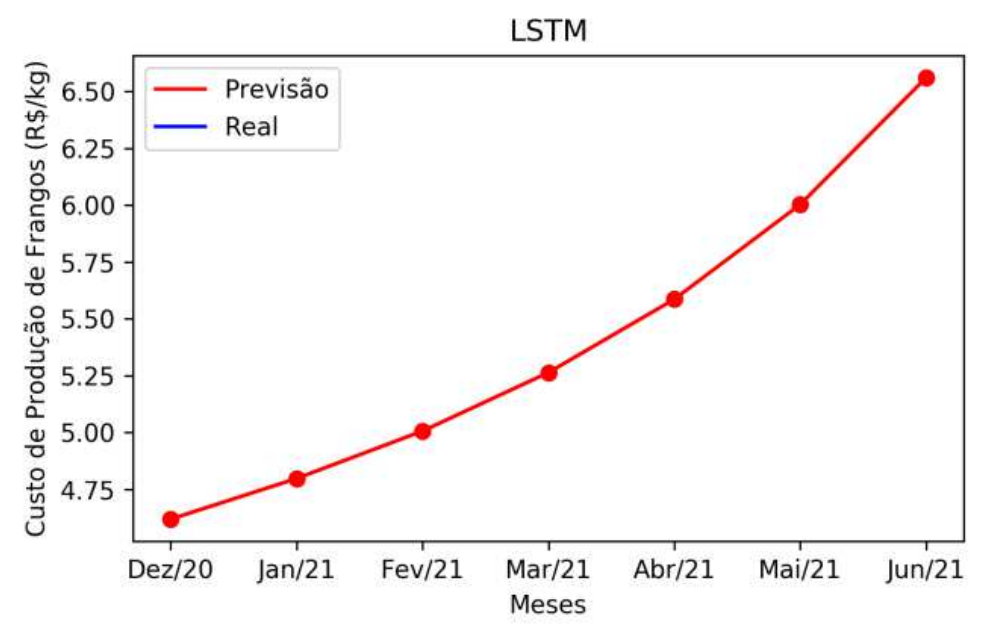

Fonte: Os autores.

\section{CONSIDERAÇÕES FINAIS}

Nesse trabalho apresentou-se uma aplicação, de modelos de redes neurais para previsão do custo de produção de frangos de corte no estado do Paraná. A série de custos de produção de frangos no período de Janeiro/2010 a Novembro/2020 (131 observações mensais), foi obtida pela Empresa Brasileira de Pesquisa Agropecuária (EMBRAPA).

Inicialmente, realizou-se uma comparação entre os modelos MLP e LSTM, ambos implementados na linguagem Python. Observou-se que os indicadores de desempenho $\left(\mathrm{r}^{2}\right.$, RMSE e MAE) obtidos pelos dois modelos apresentaram resultados muito similares.

Na sequência, observou-se, para os meses que não participaram do treinamento da rede (Janeiro à Novembro de 2020), que as previsões do modelo LSTM foram mais precisas que a do modelo MLP. Sendo que, para o modelo LSTM, as diferenças entre valores reais e preditos foram pequenas. Portanto, a proximidade entre valores preditos e reais demonstra a boa capacidade de generalização para um horizonte de onze meses, do modelo LSTM implementado neste trabalho.

Apesar do modelo LSTM apresentar resultados adequados para uma previsão de onze meses, sugere-se, para outros trabalhos de pesquisa, proceder à estimação, utilizando a biblioteca Keras, com outros modelos, tais como: os modelos BLSTM (Bidirectional Long Short-Term Memory) e GRU (Gated Recurrent Unit). 


\section{REFERÊNCIAS}

AMRAEL, S.; MEHDIZADEH, S. A.; SALARI, S. Broiler weight estimation based in machine vision an artificial neural network. British Poultry Science, vol. 58, 2017.

ABPA. Associação Brasileira de Proteína Animal. Relatório Anual Completo. Disponível em: $<$ http://abpa-br.com.br/files/ RelatorioAnual_UBABEF_2015_DIGITAL.pdf $>$. Acesso em: 12 jul. 2020.

AVISITE. Frango: o VBP de 2019 segundo os estados produtores. Disponível em: <https://www.avisite.com.br/index.php?page=noticias\&id=20598>. Acesso em: 22 abr. 2020.

BASTIANI, M.; SANTOS, J. A. A.; SCHMIDT, C. A P.; SEPULVEDA, G. P. L. Application of data mining algorithms in the management of the broiler production. Geintec, vol. 8, 2018.

CANKURT, S; SUBASI, A. Comparasion of linear regression and neural network models forecasting tourist arrivals to turkey. Eurasian Journal of Science \& Engineering, 2015.

CHEVAliER, G. The Long Short-Term Memory (LSTM) cell can process data sequentially and keep its hidden state through time. Disponível em: <https://commons.wikimedia.org/wiki/File:The_LSTM_cell.png>. Acesso em: 10 mai. 2020.

EMBRAPA. Empresa Brasileira de Pesquisa Agropecuária (EMBRAPA). Suínos e aves. Disponível em: <https://www.embrapa.br/suinos-e-aves/>. Acesso em: 2 mar. 2020.

GOMES, D. T. Redes neurais recorrentes para previsão de series temporais de memórias curta e longa. Dissertação (Mestrado em Estatística). Universidade Estadual de Campinas, Campinas, 2005.

GRAVES A. Towards end-to-end speech recognition with recurrent neural networks. In... Proceedings of the 31st International Conference on Machine Learning (ICML-14), Beijing, China, 2014.

GIL, A. C. Como elaborar projetos de pesquisa. 4 ed. São Paulo: Atlas, 2002.

HAYKIN, S. Neural networks: a comprehensive foundation. New Delhi: Pearson Prentice Hall, 2001.

JOHASEN, S. V.; BENDTSEN, J. D.; MOGENSEN, J. Broiler slaughter weight forecasting using dynamic neural network models. In... IEEE 6th International Conference (ICIEA), Tokio JP, 2019.

JÚNIOR, J. G.; BENTO, E. F.; SOUZA, A. F. Diagnóstico da realidade dos criatórios de aves na comunidade base física - Ipanguaçu/RN. Revista Holos, vol. 4, 2009.

LIMA, F. G et al. Aplicações de redes neurais na análise e concessão de crédito ao consumidor. Revista de Administração - RAUSP, vol. 44, 2009.

MESQUITA, C. M.; OLIVEIRA, R.; PEREIRA, A. C. M. Utilização de uma rede neural LSTM e teste da razão de variância para previsões em séries de ativos da Bovespa. Disponível em: <http://www.comp.ita.br/labsca/waiaf/papers/CaioMesquita_paper_11.pdf>. Acesso em: 11 abr. 2020. 
MUNTASER, J. G.; SILVA, P. S.; PENEDO, A. S. Aplicação De Redes Neurais Na Previsão De Ações Do Setor De Petróleo E Gás Da Bm\&Fbovespa. Revista FSA, vol. 14, 2017.

NELSON, M. Q.; PEREIRA, A C. M.; OLIVEIRA R. A. Stock market's price prediction with LSTM neural networks. In... International Joint Conference of Neural Networks (IJCNN). Anchorage, Alaska, 2017.

PIMENTEL, F. Curso de estatística experimental. Piracicaba: ESALQ, 2009.

PINHEIRO, T. C.; SANTOS, J. A. A.; PASA, L. A. Gestão da produção de frangos de corte por meio de redes neurais artificiais. Revista Holos, vol. 2, 2020.

PROCÓPIO, D. P; LIMA, H. J. Avaliação conjuntural da avicultura no Brasil. Research, Society and Development, vol. 9, 2020.

SANDI, A. J.; MIELE, M. Análise comparativa dos custos de produção de frangos de corte em SC e GO. Guia Gessulli da Avicultura \& Suinocultura Industrial, 2012.

SEBASTIAN, S. Performance evaluation by artificial neural network using WEKA. International Research Journal of Engineering and Technology, vol. 3, 2016.

ZAO, Z. (2017) LSTM network: a deep learning approach for short-term traffic forecast. IET Intelligent Transport Systems, vol. 11, 2017.

\section{Como Referenciar este Artigo, conforme ABNT:}

SANTOS, J. A. A. Aplicação de Redes Neurais na Previsão de Custos de Produção de Frangos de Corte no Estado do Paraná. Rev. FSA, Teresina, v.18, n. 6, art. 9, p. 169-185, jun. 2021.

\begin{tabular}{|l|c|}
\hline \multicolumn{1}{|c|}{ Contribuição dos Autores } & $\begin{array}{c}\text { J. A. A. } \\
\text { Santos }\end{array}$ \\
\hline 1) concepção e planejamento. & $X$ \\
\hline 2) análise e interpretação dos dados. & $X$ \\
\hline 3) elaboração do rascunho ou na revisão crítica do conteúdo. & $X$ \\
\hline 4) participação na aprovação da versão final do manuscrito. & $X$ \\
\hline
\end{tabular}

